\title{
Pregnancy of Un-Known Location: Need for Evolving New Protocols for DGH Units
}

\section{Rafi $J^{*}$ and Khalil $H$}

Department of Obstetrics and Gynecology, Ipswich Hospital NHS Trust, UK

*Corresponding author: Junaid Rafi, Department of Obstetrics and Gynecology, Ipswich Hospital NHS Trust, Heath Road, UK

Received: November 20, 2017; Accepted: December 12, 2017; Published: December 19, 2017

\begin{abstract}
Aims: To find out the timeline for diagnosis and outcome of pregnancy of unknown location PUL cases. To find out the approach for accuracy of diagnosis and to prevent doing unnecessary scan follow ups and BHCG tests.
\end{abstract}

Methods: Prospective observational study over one year period between January 2015 and January 2016

Results: In this study 50 patients were included and six patients were excluded as they didn't meet the criteria. The Intra-Uterine Pregnancy (IUP) was diagnosed in $28 \%(n=14)$. The suboptimal $\beta H C G$ was seen in $11 \%(n=5)$ patients, who later were diagnosed with ectopic pregnancy. The remaining $56 \%$ $(n=25 / 44)$ patients unfortunately had failing IUP, and none of them were labelled as persistent PUL.

The number of $\beta H C G$ tests undertaken in Ectopic group ranged from 3-8, compared to 2-6 times in failing IUP group whereas in IUP cohort it remained one to two tests. Similarly, we found that number of pelvic scan required for making diagnosis in three above-mentioned groups varied. Our study data revealed that each patient had 2-4 scans in Ectopic group, and 1-3 scans needed in Failing IUP.

Conclusion: To reach the final definite diagnosis / outcome in PUL cases in set time limit may not be possible in some atypical presentation of early pregnancy problems especially when the aim is to avoid un-necessary scans, follow ups and blood test without compromising the safety. Therefore we suggested a new local protocol for diagnosing and managing atypical presentations outcome will be possible from and in our study, it took 5 to 9 days durations.

Keywords: Pregnancy Un-known Location (PUL); Ectopic pregnancy

\section{Background}

Ascertaining pregnancy location is the key determinant for streamlining management for patients presenting with abdominal pain or bleeding in early pregnancy. However sometimes in spite of all efforts finding the location of gestation sac is difficult and such cases have been classified categorised as Pregnancy of Unknown Location (PUL)

As locating early pregnancy is dependent on many factors therefore incidence of PUL varies in different setup. According to recent reports the Specialist unit reported that to around $8-10 \%$ vs. 8-31\% in non-specialist Unit [1].

The lack of well accepted consensus for diagnostic criteria for PUL is reflected by differences in the management strategies of PUL.

The possibility of missing an ectopic pregnancy in the course of PUL management may lead to higher rate of maternal mortality and morbidity, likelihood of requiring a surgical intervention.

It can adversely affect patients' and health professionals' confidence for any management of PUL.

The main concern remains the potential interventions of a PUL case which may be a viable intrauterine pregnancy.
PUL case reports in UK makes it evident that most hospitals are lacking a clear and predictable PUL managing tool. However the PUL outcome data is very encouraging from hospitals that have developed clear PUL care pathways incorporating reliable diagnostic criteria.

Only few UK units have opted their PUL management based on a clear predictive tool including widely accepted hormone tests.

We conducted a prospective study to review PUL managed cases and aimed to propose a PUL management pathway and thereby reducing variation.

\section{Aim}

The aim was to find out the timeline for diagnosis and outcome of PUL cases and to propose the prompt and effective approach for PUL diagnosis. Also wanted to find out the pattern and frequency of scans and BHCG in PUL cases.

\section{Criteria}

PUL cases were identified if there was no evidence of intrauterine or extra uterine pregnancy on first transvaginal ultrasound scan (5-6 week gestation) in women with a positive pregnancy test.

\section{Methods}

This was a prospective observational study, including all patients 


\section{Incidence}

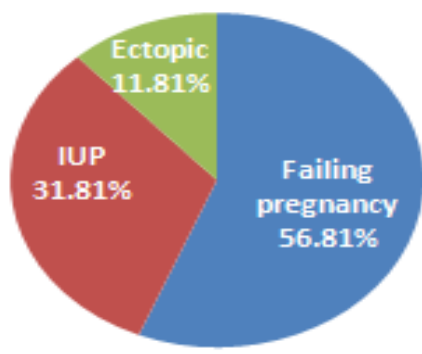

Figure 1: Outcome PUL cases.

Table 1: Frequency of $\beta \mathrm{HCG}$ and Transvaginal ultrasound scans in each patient.

\begin{tabular}{|c|c|c|c|}
\hline $\begin{array}{c}\text { Outcome } \\
\text { Group }\end{array}$ & $\begin{array}{c}\text { No. scans / } \\
\text { patient }\end{array}$ & $\begin{array}{c}\text { No. of } \beta \text { HCG / } \\
\text { Patient }\end{array}$ & $\begin{array}{c}\text { Mean time of diagnosis } \\
\text { established (Days) }\end{array}$ \\
\hline $\begin{array}{c}\text { Failing } \\
\text { Pregnancy }\end{array}$ & $1-3$ & $2-6$ & $2-32$ \\
\hline Ectopic & $2-4$ & $3-8$ & $6-21$ \\
\hline IUP & $1-2$ & $1-2$ & $2-14$ \\
\hline
\end{tabular}

attending EGAU (Early Gynaecology Assessment Unit) who were diagnosed as PUL between January 2015 and January 2016 at District General Hospital, and cases were followed up for object analysis of outcome.

\section{Results}

Fifty patients who attended EGAU were included in this PUL study. Six patients were excluded as they didn't meet the criteria of PUL study protocol. The $28 \%(\mathrm{n}=14)$ found to have Intra-Uterine Pregnancy (IUP).

The suboptimal $\beta$ HCG rise was seen in $11 \%(n=5)$ patients, who later were diagnosed with ectopic pregnancy. The remaining 56\% ( $n=25 / 44)$ patients failing IUP and none of them were labelled as persistent PUL (Figure 1).

The serum $\beta$ HCG assessments were undertaken 3-8 times before reaching diagnosis in Ectopic group whereas in Failing IUP group serum $\beta$ HCG was done 2-6 times and in IUP cohort it remained one to two tests.

Our data revealed that number of scans required for each patient in above mentioned three groups varied. For ectopic group patients had 2-4 scans, and patients in failing IUP \& IUP group required an average 1-3 pelvic scans (Table 1).

In cases which required more than three $\beta \mathrm{HCG}$ the management was discussed with consultant on call for further follow up. The analysis of ectopic pregnancy group revealed that suboptimal rise in serum $\beta$ HCG and ultrasound scan findings were the main factors considered leading to the diagnosis of ectopic.

All patients in ectopic group remained hemodynamically stable. The mean time to establish diagnosis in ectopic group was 6-21 days, and serum $\beta$ HCG discriminatory zone values ranged (1500$2400 \mathrm{IU} / \mathrm{L}$ ). One case diagnosed as ectopic pregnancy on 21 st day of reporting EGAU, while the serum $\beta$ HCG values were $<1500 \mathrm{IU} / \mathrm{L}$
Table 2: Validity of $\beta H C G$ level ratio @ 48 h/ $0<0.87$ as predictive tool for failing pregnancy.

\begin{tabular}{|c|c|}
\hline BHCG $48 \mathrm{hr} / 0$ hr Ratio & $\begin{array}{c}\text { Number of cases out of total } \\
\text { failing pregnancy group }(\mathrm{n} / 25)\end{array}$ \\
\hline $\begin{array}{c}\text { Number of cases }(\mathrm{n}) \text { in failing pregnancy } \\
\text { group where } \beta \mathrm{HCG} \text { ratio was }<0.87\end{array}$ & $14 / 25(56 \%)$ \\
\hline $\begin{array}{c}\text { Number of cases }(\mathrm{n}) \text { in failing pregnancy } \\
\text { group where } \beta \mathrm{HCG} \text { ratio was }>0.87\end{array}$ & $10 / 25(40 \%)$ \\
\hline
\end{tabular}

Table 3: Validity of $\beta \mathrm{HCG}$ level ratio @ 48h/0h $>2.00$ as predictive tool for a viable Intrauterine pregnancy (IUP).

\begin{tabular}{|c|c|}
\hline$\beta H C G$ 48hr/0 hr Ratio & $\begin{array}{c}\text { Number of cases out of total } \\
\text { IUP group }(n / 25)\end{array}$ \\
\hline $\begin{array}{c}\text { Number of cases }(n) \text { in early IUP group where } \\
\beta H C G \text { ratio was >2 }\end{array}$ & $9 / 14(64 \%)$ \\
\hline $\begin{array}{c}\text { Number of cases }(n) \text { in early IUP group where } \\
\beta H C G \text { ratio was > 1.6-2 }\end{array}$ & $5 / 14(35 \%)$ \\
\hline
\end{tabular}

and patient had Laparoscopic salpingectomy. The other such case managed with Methotrexate where $\beta$ HCG was $<500 I U / L$.

\section{Discussion}

The term 'Pregnancy of Unknown Location' (PUL) is referred when there is no conclusive evidence of either intra- or extra uterine pregnancy or retained products of conception on transvaginal ultrasound, despite a positive pregnancy test [2].

It is a well-known fact that laparoscopy has a false negative rate of $3-4 \%$ when undertaken done too early. It has been reported that $5 \%$ false positive rate of laparoscopy are due to the presence of retrograde uterine bleeding in miscarriage which can be misinterpreted as tubal ectopic pregnancy especially ultrasound reported presence of free fluid in pelvis.

Many reported that there are more variations in pelvic scan reports at smaller units. The less consistency in scan report quality in non-specialist unit has been recognised as issue.

This trend was seen in our study as well requiring up to four scan in some patients of ectopic group and three scans in failing IUP group.

In spite NICE Guideline no CG154 [3] recommendation that management with Methotrexate can be considered at $\beta$ HCG levels up to of 3000IU/L in hemodynamically stable patients but our unit proposed $\beta$ HCG level of 500-2400 IU/L; it can be argued that it might be due to less consistent scans reports.

As there are still no conclusive RCT (randomised controlled trials) published compare different management strategies for PUL. There seemed a clinical heterogeneity between the studied populations.

Therefore, the need for developing management strategies for PUL and other atypical early pregnancy problems each DGH unit assumes great significance.

It also highlights to revisit the policy to adhere to specific models extrapolated from studies conducted in relatively bigger specialist units where possible best expertise and equipment are available. It is rather advisable to develop management PUL protocol; taking into the consideration demographics of population and available facilities and expertise. We proposed a new protocol shown below based on findings from our study (Figure 2).

However this audit showed (Table 1) that time to finalise a 


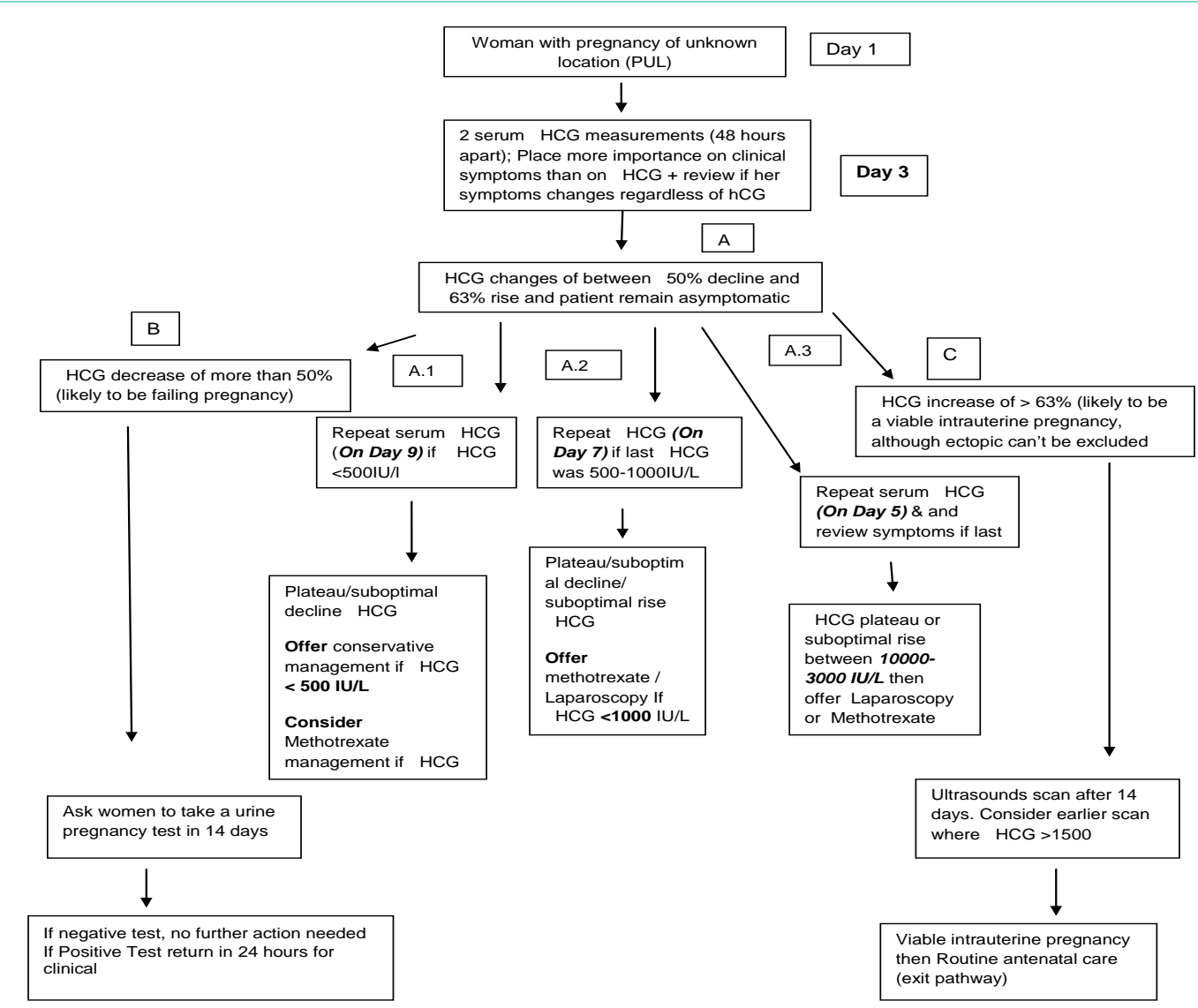

Figure 2: Pregnancy of Unknown location Management Protocol.

Pathway A1: For Plateau or suboptimal decline $\beta$ HCG values $<500 \mathrm{IU} / \mathrm{L}$ : Total duration of Initial presentation to treatment offered comprise of 9 days.

Pathway A2: For Plateau or suboptimal decline $\beta$ HCG values 500IU/L-1000IU/L: Total duration of Initial presentation to treatment offered comprise of 7 days.

Pathway A3: For Plateau BHCG values of $>1000 \mathrm{IU} / \mathrm{L}$ and above: Total duration of Initial presentation to treatment offered comprise of 5 days.

Caution: At the moment Methotrexate is only recommended for established diagnosis of Ectopic pregnancy. Role of Methotrexate in management of PUL is not well defined and should be offered with caution and after informed consent of patient.

diagnosis was anywhere between 2-32 days for failing pregnancy and ranged between 6-21 days for ectopic pregnancy. It can be argued that setting a target (time limit) to reach definitive diagnosis may not be achievable in all patients but in new proposed protocol total duration from initial presentation to final treatment offered will be 9,7 and 5 days in Pathway A.1, A.2 and A.3 respectively 4 days in pathway $\mathrm{A}$ and $\mathrm{B}$ respectively (Figure 2).This pathway (Figure 2) follows the same flow as per NICE Assessment of pregnancy of un known location interactive flow chart (6) but differs from point $\mathrm{A}$ to further into A1, A2 and A3 whereas pathway B and pathway C; are same as NICE Assessment of pregnancy of un known location interactive flow chart (6).

As we aimed by conducting this study to propose best approach of such cases by improving the accuracy of diagnosis and to prevent doing unjustified scans and $\beta$ HCG. It is important to clarify which cases should be labelled as PUL; there are number of different models designed to define and classify PUL management based on various mathematical calculations, including $\beta$ HCG with or without progesterone levels.

While proposing PUL management pathways in our department we considered various other models for their suitability to be adopted.
It was realized that two such protocols seemed more pragmatic to implement safely in our unit for the prediction of failing group and early intrauterine pregnancy group.

One was proposed by Condous et al [4] that the serum $\beta$ HCG ratio at $48 \mathrm{hr}$ : $0 \mathrm{hr}<0.87$ promises an optimal test for the prediction of failing pregnancy. The other model was suggested by Bignardi $\mathrm{T}$ et al [5] who concluded that $\beta \mathrm{HCG}$ ratio $48 \mathrm{hr}: 0 \mathrm{hr}>2.00$ points toward increased chances for a viable IUP.

It can be demonstrated in our study that serum $\beta$ HCG ratio $<0.87$ (Table 2) can easily and safely be used for predicting failing pregnancy in PUL labelled cases. Similarly, serum $\beta$ HCG ratio $>2.0$ (Table 3) can be helpful in predicting ongoing early intrauterine pregnancy. However instead of adopting these two predicting models we proposed to follow Pathway B and Pathway C from NICE Assessment of pregnancy of unknown location interactive flow chart (6) for failing pregnancy and IUP group respectively.

We argue the fact that PUL is such an entity that may need keep evolving new protocols locally in DGH units and testing and proving the validity of predictor tools provide confidence in setting up new protocols for the individualised care plan for patients with the expertise. 


\section{Strengths and Limitations}

This was a small but prospective study conducted in District General Hospital but a sample size of more than 40 was taken to ensure that the sample mean is approximately normally distributed. The inferences drawn may not applicable nationally for National guidelines but hopefully at DGH units in UK can incorporate the suggestions in their departmental protocols based on the assumption that early pregnancy assessment units are similar in terms of patient load, staff expertise and equipment.

\section{Conclusion}

To reach the final definite diagnosis / outcome in PUL cases in set time limit may not be possible in some atypical presentation of early pregnancy problems especially when the aim is to avoid unnecessary scans, follow ups and blood test without compromising the safety. Therefore we suggested a new local protocol for diagnosing and managing atypical presentations outcome will be possible from and in our study, it took 5 to 9 days durations This variation is due to atypical presentation of PUL cases and DGH units may introduce pathways which that may be little different from national or tertiary unit protocols. The key message is to keep on reviewing and auditing their results to ensure safety.

\section{Recommendations}

- Introduction of local pathways where initial presentation to final treatment will be offered 9 days (serum $\beta$ HCG $<500 \mathrm{IU} / \mathrm{L}$ ), 7 days (serum $\beta$ HCG 500-1000IU/L) and 5 days where BHCG will be $>1000 \mathrm{IU} / \mathrm{L}$. It may help reducing unnecessary follow ups and may streamline management of difficult scenarios of plateauing serum $\beta$ HCG.
- Re-audit in a year's time to review and audit the results to ensure safety.

- Use of methotrexate in selective hemodynamically stable patients and given after patient counseling and careful consideration that possibility of early viable pregnancy is ruled out.

\section{References}

1. Sagili H, Mohamed K. Pregnancy of unknown location: an evidence-based approach to management. The Obstetrician \& Gynaecologist. 2008; 10: 224 230.

2. Royal College of Obstetricians and Gynaecologists. The Management of Early Pregnancy Loss. Green- top Guideline No. 252006 London: RCOG. 2006.

3. Ectopic Pregnancy and miscarriage: Diagnosis and initial management in early pregnancy of ectopic pregnancy and miscarriage (CG154). 2012.

4. Condous G, Van Calster B, Kirk E, Timmerman D, Van Huffel S and Bourne T. Prospective cross-validation of three methods of predicting failing pregnancies of unknown location Hum. Reprod. 2007; 22: 1156-1160.

5. Bignardi G, Condous D, Alhamdan E, Kirk B, Van Calster B, Van Huffel $S$, et al. The HCG ratio can predict the ultimate viability of the intrauterine pregnancies of uncertain viability in the pregnancy of unknown location population. Hum. Reprod. 2008; 23: 1964-1967.

6. NICE Assessment of pregnancy of unknown location interactive flow chart.
Austin J Obstet Gynecol - Volume 4 Issue 5 - 2017 Submit your Manuscript | www.austinpublishinggroup.com Rafi et al. (C) All rights are reserved
Citation: Rafi J and Khalil H. Pregnancy of Un-Known Location: Need for Evolving New Protocols for DGH Units. Austin J Obstet Gynecol. 2017; 4(5): 1088. 